\title{
Photodynamic treatment induces cell death by apoptosis or autophagy depending on the melanin content in two B16 melanoma cell lines
}

\author{
AGNÈS SPARSA $^{1,2}$, SOLENN BELLATON ${ }^{1}$, THOMAS NAVES ${ }^{1}$, MARIE-ODILE JAUBERTEAU ${ }^{1}$, \\ JEAN-MARIE BONNETBLANC ${ }^{2}$, VINCENT SOL ${ }^{3}$, MIREILLE VERDIER ${ }^{1}$ and MARIE-HÉLÈNE RATINAUD ${ }^{1}$ \\ ${ }^{1}$ EA 3842 Cellular Homeostasis and Pathology, Faculty of Medicine, University of Limoges, \\ 87025 Limoges Cedex; ${ }^{2}$ Department of Dermatology, University Hospital Dupuytren, 87042 Limoges Cedex; \\ ${ }^{3}$ Chemistry Laboratory of Natural Substances, Faculty of Sciences, 87060 Limoges Cedex, France
}

Received July 19, 2012; Accepted September 17, 2012

DOI: $10.3892 /$ or.2012.2190

\begin{abstract}
Photodynamic therapy (PDT) is now a well-established treatment modality for cutaneous carcinomas and is based on the administration of a light-activated drug followed by illumination of the pathological area. The treatment of metastatic melanoma remains a therapeutic challenge. To define the possible role of melanin in relative phototoxicity of 5-aminolevulinic acid (5-ALA), a photosensitizer used in PDT in vivo, we studied cell death in two variants (with or without melanin, B16F10 and B16G4F cells, respectively) of a melanoma cell line. Concentrations of 5-Ala up to $10 \mathrm{mM}$ induced similar cytostatic effects in the B16G4F and B16F10 cells. PDT and high 5-ALA concentrations induced photocytotoxicity in both melanoma cell lines (at $10 \mathrm{mM}$ for B16F10 cells and at $5 \mathrm{mM}$ for B16G4F cells). Cell death corresponded to p53-dependent apoptotic signaling in pigmented B16F10 cells, whereas an autophagic response leading to a caspase-independent death was detected in nonpigmented B16G4F cells. Therefore, the PDT-induced cell death pathway appeared to correlate with melanin synthesis capacity in melanoma cells. To reduce the cytotoxicity of 5-ALA without irradiation, a low drug concentration could be used. Consequently, in combination with current therapeutics, a moderate concentration of 5-ALA and PDT may constitute a supplementary promising approach to eliminate metastatic melanoma.
\end{abstract}

Correspondence to: Dr Mireille Verdier, EA 3842 Cellular Homeostasis and Pathology, Faculty of Medicine, University of Limoges, 2 rue Dr Marcland, 87025 Limoges Cedex, France

E-mail: mireille.verdier@unilim.fr

Key words: melanoma, autophagy, photodynamic therapy, 5-aminolevulinic acid, cell death, melanin, pan-caspase, p53, PUMA, beclin, LC3, cathepsin B

\section{Introduction}

Photodynamic therapy (PDT) has been used in clinical application for many years, either systemically, or locally or topically applied to a patient bearing a lesion (cancerous or not). PDT with systemic photosensitizers has been used to treat a range of internal malignancies (lung and brain cancers) and for skin cancers (1). This cancer treatment is approved by many countries for the treatment of non-melanotic skin cancers such as basal cell carcinomas and Bowen's disease. PDT does not seem to be an appropriate treatment of melanotic melanoma in vivo, and several reasons can justify this low use. Almost all melanomas overexpress the classical ATP-binding cassette transporters (ABC protein family), leading to drug release into the extracellular medium and to the resistance by tumor cells. The antioxidant activity of melanoma is much higher than observed in other skin cancers (basal and squamous carcinomas) reducing the cytotoxic effect of ROS generated by PDT (2). Nearly all photosensitizers (PS) show a significant absorbance in ultraviolet (UV) wavelengths, while melanin absorbs a large portion of the light intended to activate PS. The light absorption by pigment strongly reduces its penetration into the tumor. Studies have been carried out with pigmented or amelanotic melanoma cells suggesting that amelanotic cells may be more susceptible to PDT (2). Among the molecules used in PDT, 5-aminolevulinic acid (5-ALA) is not itself a photosensitizer and instead it is used as a prodrug leading to the accumulation of protoporphyrin IX in the mitochondria inducing damage and subsequently cell death after light irradiation (3).

It is well-known that exposure of a photosensitizing agent to appropriate light leads to a photophysical reaction and the production of reactive oxygen species (ROS). These constitute the main driving force to initiate three types of cell death (necrosis, apoptosis or autophagy). Nevertheless, the signaling pathways involved in PDT-mediated cell death are not completely understood.

In this study, we examined the photocytotoxicity of 5-ALA on two variants of melanoma cell lines (pigmented B16F10 and amelanotic B16G4F cells), the cell death and autophagic 
pathways were investigated. One day after the induction of PDT with 5-ALA, the pan-caspase activity differed between the two melanoma cell lines. The recruitment of p53 and PUMA led to a caspase-dependent cell death in the pigmented cells whereas an autophagic cell death was activated in the non-pigmented (amelanotic) melanoma cells.

\section{Materials and methods}

Source and preparation of the photosensitizer. 5-ALA (5- $\delta$-aminolevulinic acid hypochloride) was obtained from Sigma-Aldrich Chimie (Saint-Quentin Fallavier, France) and prepared as a stable $30 \mathrm{mM}$ stock solution (stored in aliquots at $-80^{\circ} \mathrm{C}$ ) from the lyophilized powder and was dissolved in Dulbecco's modified Eagle's medium (DMEM, InvitrogenFisher, Illkirch, France) containing $10 \%$ fetal bovine serum (FBS) (Invitrogen-Fisher). Other reagents are specified below.

Cell lines and culture conditions. The B16F10 pigmented melanoma cells were a gift from Dr Perron (Oncology Center, Toulouse, France). The amelanotic B16G4F melanoma cells (without Mc1r expression, the receptor of $\alpha$-melanocyte stimulating hormone) were kindly provided by Professor A. Oulmouden (UMR INRA 1061, Limoges, France). Cells were grown to confluence in DMEM containing 10\% FBS and antibiotics (100 U/ml penicillin and $100 \mu \mathrm{g} / \mathrm{ml}$ streptomycin) in a humidified $5 \% \mathrm{CO}_{2}$ atmosphere at $37^{\circ} \mathrm{C}$. The culture medium was renewed every 3 days. Cells were subcultured by dispersal with trypsin-EDTA and replated at $2 \times 10^{5} / \mathrm{ml}$.

Light source. Exposure of melanoma cells to light was performed using either a red lamp composed of fluorescent tubes or light emitting diodes (LED) (Aktilite ${ }^{\mathrm{TM}} 128$ Lamp, Galderma). The light fluence rate used by the red lamp was $37 \mathrm{~mW} / \mathrm{cm}^{2}$ at $631 \mathrm{~nm}$ on the surface of the plate.

Irradiation protocol. Cells were seeded at $10^{5}$ cells $/ \mathrm{ml}$ in 96-well plates after trypsinization. After $24 \mathrm{~h}$, the cells were washed twice with PBS and incubated with different concentration of 5-ALA $(0,2.5,5,10$ or $20 \mathrm{mM})$ for $2 \mathrm{~h}$ with medium, in darkness and in a humidified $5 \% \mathrm{CO}_{2}$ atmosphere at $37^{\circ} \mathrm{C}$. The cells were then irradiated with the Aktilite Lamp $^{\mathrm{TM}}$ at room temperature $\left(25^{\circ} \mathrm{C}\right)$. The exposure time was adjusted to obtain $37 \mathrm{~J} / \mathrm{cm}^{2}$ fluence (corresponding to $\sim 8 \mathrm{~min}$ ). In previous experiments, during cell illumination the temperature monitored at the top of the microplate was unchanged. Two controls were realized, one 96-well plate without photosensitizer and one with photosensitizer and without irradiation.

Cytotoxic and photocytotoxic studies. Cytotoxicity and photocytoxicity were measured using the XTT(3-(4,5-dimethylthiazol-2-yl)-2,5-diphenyltetrazolium bromide) colorimetric assay (Cell Proliferation Kit II, Roche Diagnostics, Meylan, France). Optical density is proportional to relative mitochondrial dehydrogenase activity and reflects cell viability. Three hours after the addition of 5-ALA, assays were performed at 0,24 and $48 \mathrm{~h}$ either without irradiation or after the end of the irradiation. At the time of counting, $50 \mu \mathrm{l}$ of DMEM without phenol red-XTT solution $(0.3 \mathrm{mg} / \mathrm{ml})$ was added and incubation was carried out for $3 \mathrm{~h}$ at $37^{\circ} \mathrm{C}$. Optical density (OD) at $490 \mathrm{~nm}$ was determined (the OD reference at $690 \mathrm{~nm}$ was subtracted) in an MRX II (Dynex) absorbance reader. Cytotoxicity was evaluated by the OD ratio of treated vs. non-treated cells. Each value of XTT assay was obtained from cultures in triplicate.

Pan-caspase activity assay. Both melanoma cell lines were plated in a 24 -well dish (Nunc) at $25 \times 10^{4}$ cells/well. After a 24-h culture, 5-ALA was added or not to the cells and irradiation was carried out according to the protocol. After incubation, cells were pooled and lysed in Chaps buffer. The assays were performed as previously described (4). Peptide substrates (Bachem, Germany) for pan-caspase (Z-VAD-AMC) were added to each well to a final concentration of $5 \times 10^{-5} \mathrm{M}$. Pan-caspase (Z-VAD-CHO) inhibitors at $2.5 \mathrm{mM}$ were added $30 \mathrm{~min}$ before the substrate. The assay plates were incubated at $37^{\circ} \mathrm{C}$ for $2 \mathrm{~h}$ and fluorescence was measured with a microplate reader (Twinkle LB 970, Berthold, France), then pan-caspase activities were expressed in relative fluorescence units (RFU). Background fluorescence was determined in wells containing Z-VAD-AMC assay mixture and substrate without cell lysates, and pan-caspase activity was expressed in relative units.

Western blotting. Protein lysates $(30 \mu \mathrm{g})$ from ALA-treated or control B16F10 and B16G4F cells $\left(2 \times 10^{6}\right.$ cells/dish) were separated by SDS-PAGE. After electrophoretic migration, proteins were electroblotted onto cellulose membranes. After the blocking step (TBS-1X containing $0.1 \%$ Tween 20 and 5\% non-fat dry milk) membranes were incubated with primary specific antibodies, recognizing the following antigens: rabbit anti-PUMA obtained from Cell Signaling (Ozyme, France), anti-cathepsin B (CA10) purchased from Abcam (France); tubulin (clone TU-02), beclin-1 (E-8) and MAP-LC3-II (H-50) and p53 (Bp53-12) obtained from Santa Cruz Biotechnology (Tebu-bio, France). They were revealed with appropriate secondary HRP-conjugated antibodies purchased from DakoCytomation (France). Blots were developed with Immobilon Western chemiluminescent HRP substrate from Millipore and analyzed with the G-Box (Ozyme). Each set of experiments was repeated independently for at least 3 times.

\section{Results}

Different levels of photocytotoxicity of 5-aminolevulinic in B16F10 and B16G4F murine melanoma cell lines. A time kinetic (0-48 h) study of cytotoxicity, measured with the XTT assay, corresponding to relative dehydrogenase activity (RDA), was carried out. Two series of experiments without or after red illumination were performed using 5, 10 and $20 \mathrm{mM}$ of 5-ALA, respectively for the pigmented B16F10 melanoma cell line and with $2.5,5$ and $10 \mathrm{mM}$ of 5-ALA for the amelanotic B16G4F melanoma cell line (Fig. 1). In both cell lines a similar cytostatic effect occured in relationship with 5-ALA concentrations. Nevertheless, after $48 \mathrm{~h}$, the relative dehydrogenase activity increased in the non-irradiated cells. At $20 \mathrm{mM}$ 5-ALA in the B16F10 cells the slowing of growth was not effective.

After photodynamic treatment (PDT) a moderate and transitional reduction in RDA was observed when compared to control cells at low 5-ALA concentrations; $5 \mathrm{mM}$ for pigmented B16F10 and $2.5 \mathrm{mM}$ for non-pigmented B16G4F 

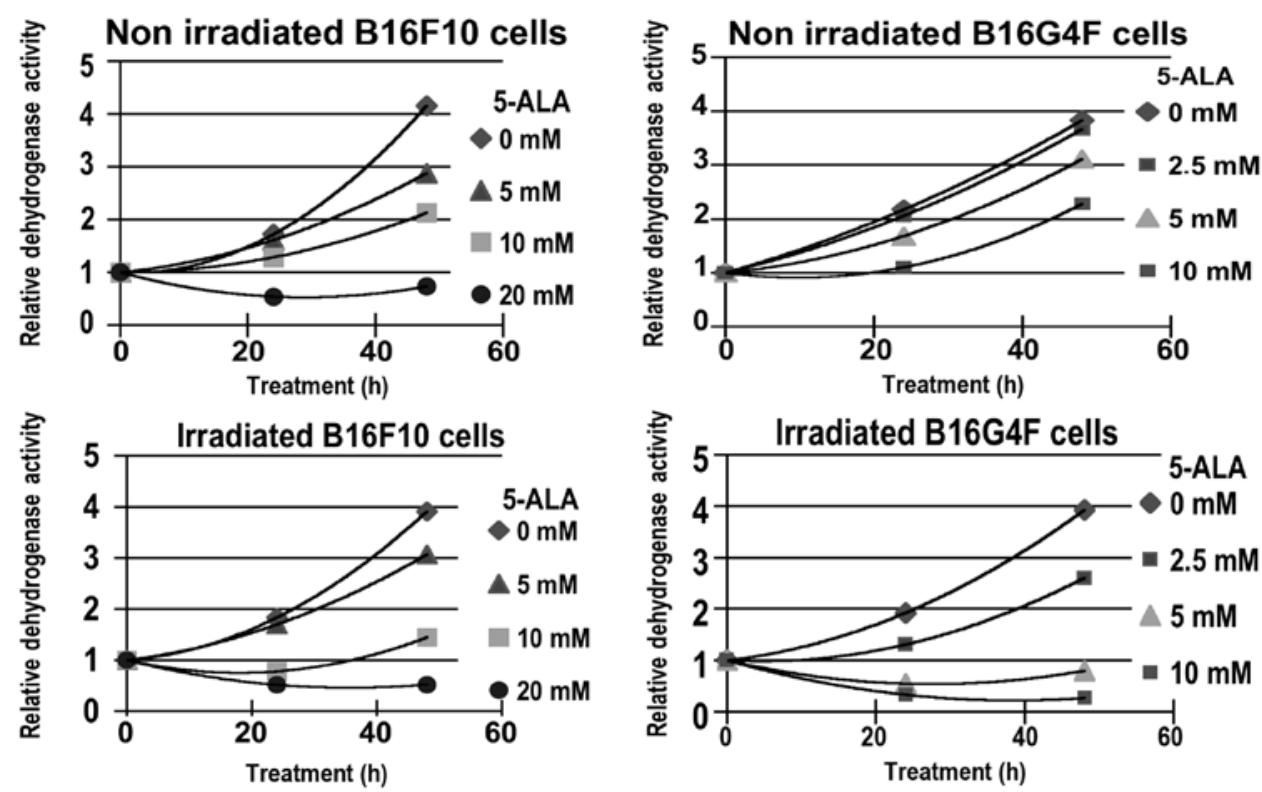

Figure 1.5-ALA and PDT induce photocytotoxicity and cytotoxicity in two melanoma murine cell lines. A cytotoxic effect appeared similar in relation to 5-ALA concentrations both in the pigmented B16F10 melanoma cell lines and in the amelanotic B16G4F melanoma cell line. The photocytotoxicity of 5-ALA appeared more intense in the amelanotic B16G4F cell line than that in the pigmented B16F10 cells. Cytotoxicity (with or without illumination) was measured using an XTT colorimetric assay proportional to the relative mitochondrial dehydrogenase activity which reflected cell viability. Three hours after the addition of 5-ALA (0-20 mM), assays were performed at 0,24 and $48 \mathrm{~h}$ either without irradiation or after the end of the irradiation.

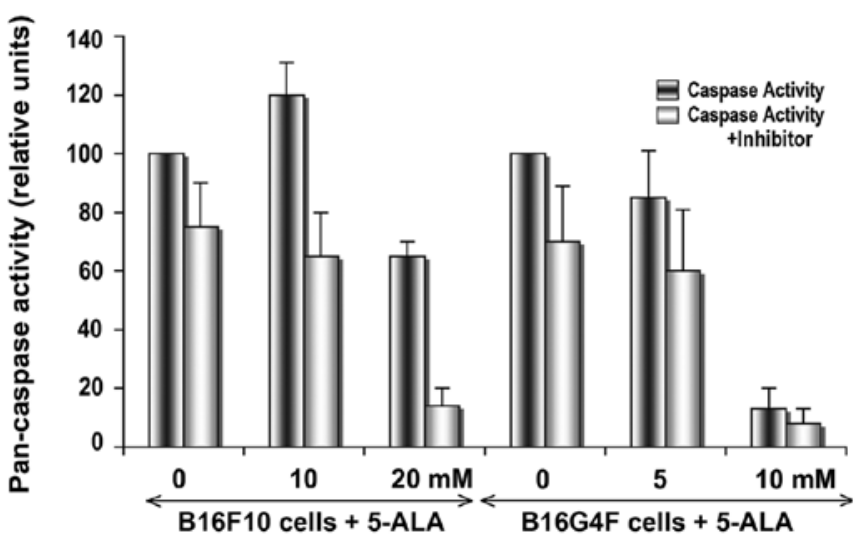

Figure 2. 5-ALA and PDT induce a caspase-dependent- and caspaseindependent cell death in the two melanoma murine cell lines. A $10-\mathrm{mM}$ concentration of 5-ALA with PDT treatment increased the pan-caspase activity in the pigmented B16F10 melanoma cells, whereas $5 \mathrm{mM} 5$-ALA did not modify the enzymatic activity of the amelanotic B16G4F cell line The disappearance of pan-caspase activity was observed for higher 5-ALA concentrations (20 mM for B16F10 cells and $10 \mathrm{mM}$ for B16G4F cells) and corresponded to cell lysis. The total caspase activity (evaluated with a fluorometer and expressed in relative units) was determined for both melanoma cell lines $24 \mathrm{~h}$ after various 5-ALA concentrations and PDT, with a peptide substrate recognized by all caspases (pan-caspase) together and in the presence of a specific inhibitor (ZVAD-CHO).

cells, respectively (Fig. 1). For higher 5-ALA concentrations (10 mM for B16F10 and $5 \mathrm{mM}$ for B16G4F cells, respectively) a significant photocytotoxicity was observed.

Pan-caspase activity in B16F10 and B16G4F cell lines after photodynamic treatment. In order to detect the apoptotic process after a 24 -h cell irradiation, total caspase activity was measured with a peptide substrate recognized by all caspases (pan-caspase), and compared with the same experiments in the presence of a specific inhibitor (ZVAD-CHO). In pigmented B16F10 cells, the pan-caspase activity was significantly increased following addition of $10 \mathrm{mM} 5$-ALA in comparison with the action in the control cells (Fig. 2). In the B16G4F cells, no significant change was observed between the control conditions and cells after PDT in combination with $5 \mathrm{mM}$ 5-ALA. At high 5-ALA concentrations $(20 \mathrm{mM}$ for B16F10 cells and $10 \mathrm{mM}$ for B16G4F cells, respectively), a drastic disappearance in caspase activity was detected corresponding to cell death at $24 \mathrm{~h}$.

Expression of p53 and PUMA in B16F10 and B16G4F cell lines after $P D T$. We studied the well-known tumor suppressor p53 protein involved in cell cycle control and apoptosis induction upon stress stimuli (Fig. 3). A significant p53 expression was observed in the control B16F10 cell line; $24 \mathrm{~h}$ after PDT treatment, the p53 signal was enhanced with the addition of $10 \mathrm{mM}$ 5-ALA but its level was reduced in the presence of $20 \mathrm{mM}$ 5-ALA. In contrast, no or a faint p53 expression was observed in the non-pigmented B16G4F cells regardless of the conditions (control, 5 and $10 \mathrm{mM}$ 5-ALA treatment). Results of the PUMA analysis, one of the p53 transcriptional targets implicated in executive apoptosis, confirmed the findings concerning p53. PUMA expression in the pigmented B16F10 cells was correlated to that of p53 and in non-pigmented B16G4F cells only a faint PUMA band was detected.

Expression of beclin-1, LC3-I, LC3-II and cathepsin-B after photodynamic treatment with 5-ALA. We studied the eventual activation of an autophagic signaling, through the expression of beclin-1, a regulator activated upstream, LC3-I 


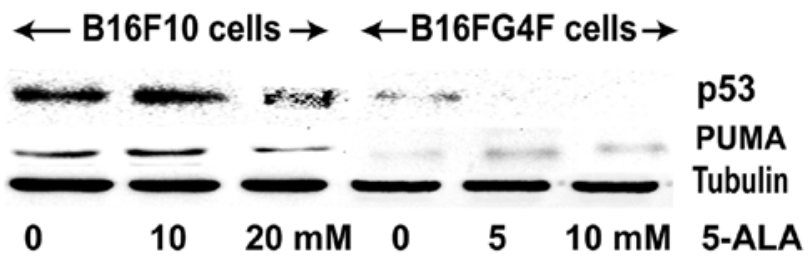

Figure 3. Western blot analysis of p53 and PUMA expression after PDT with 5-ALA in B16F10 and B16G4F melanoma cells. An increase in p53 and PUMA expression was observed $24 \mathrm{~h}$ after $10 \mathrm{mM}$ 5-ALA and PDT treatment of B16F10 melanoma, but no significant changes in expression in the B16G4F cell line was detected regardless of the condition. Cell lysates were realized $24 \mathrm{~h}$ after 5-ALA and light exposure, they were separated by SDS-PAGE and analyzed by western blotting with appropriate antibodies.

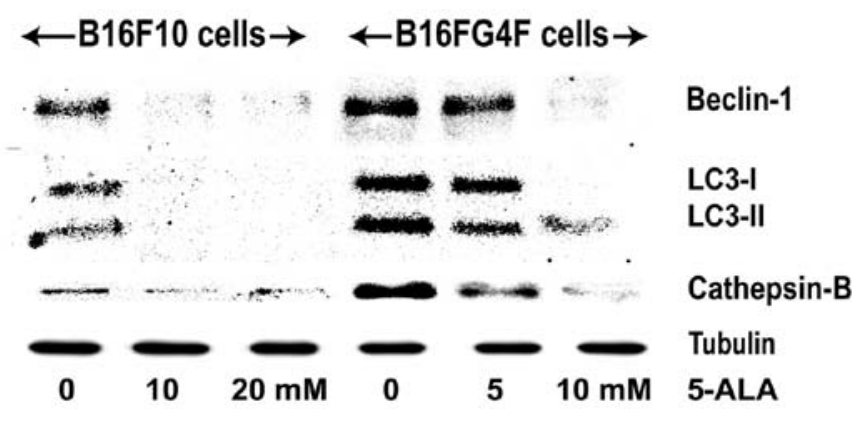

Figure 4. Western blot analysis of beclin-1, LC3-I, LC3-II and cathepsin-B expression after PDT with 5-ALA in B16F10 and B16G4F melanoma cells. Slight changes in beclin-1, LC3-I, LC3-II and cathepsin B expression were observed following 5-ALA and PDT treatment of B16F10 melanoma, whereas in the amelanotic B16G4F cell line autophagic markers were clearly enhanced after $5 \mathrm{mM}$ 5-ALA and PDT, but were significantly reduced for the photocytotoxic $10 \mathrm{mM}$ 5-ALA treatment. Cell lysates were realized $24 \mathrm{~h}$ after 5-ALA and light exposure, they were separated by SDS-PAGE and analyzed by western blotting with appropriate antibodies.

and LC3-II which is implied in the nucleation of autophagosome and a lysosomal protein cathepsin B which concludes the autophagic process (Fig. 4). In control pigmented B16F10 cells, a moderate signal was detected for both beclin-1, LC3-I and II and cathepsin-B, which disappeared in the treated cells. Conversely, their expression was significantly increased in the control non-pigmented B16G4F cells and was reduced at a higher 5-ALA concentration (10 mM).

\section{Discussion}

Photodynamic therapy (PDT) is now a well-established treatment modality for cutaneous carcinomas camptothecin $(1,5)$, but melanotic melanomas generally have a poor response to PDT owing to several characteristics that confer chemoresistance such as high antioxidant activity (2). The presence of melanin permits the absorption of UV and spectral light by pigmented melanoma, reducing photosensitizer excitation. The cell death pathway induced appears to differ in both according to photosensitizer, the cell type and in the case of melanoma with the presence of melanin. Thus, we studied the type of signaling pathway induced (apoptosis and autophagy) by 5-ALA, a physiologic compound currently used in vivo for various types of tumors, excited with a red illumination on the well-known mouse melanoma cell line B16F10 and its amelanotic variant B16G4F cells.

As expected, the 5-ALA molecule showed a similar and moderate toxicity in both pigmented and amelanotic cells. At almost all 5-ALA concentrations and after a 48-h treatment, cell growth was reinitiated concomitant with a recycling process. After $24 \mathrm{~h}$, the 5-ALA photocytotoxicity varied as a function of the presence of melanin in the melanoma cells, and when the decrease in mitochondrial activity was moderate due to a repair process, this permitted a partial restoration of cell growth after $48 \mathrm{~h}$. The slight reduction in dehydrogenase activity in the presence of low 5-ALA concentrations was noted in the two melanoma cell lines (pigmented or not), then the restart of cell growth was observed at $48 \mathrm{~h}$, which was consistent with an autophagic process. Due to the high reactivity of photogenerated ROS, autophagy is initiated to remove oxidative damaged organelles such as mitochondria which are targets of 5-ALA $(5,6)$. The $10 \mathrm{mM}$ concentration of 5-ALA induced photocytotoxicity in B16F10 cells slightly weaker than that observed in the B16G4F amelanotic cells treated with $5 \mathrm{mM}$ 5-ALA. Several studies have previously demonstrated that the presence of melanin in melanoma reduces drug photocytotoxicity. Ma et al (7) showed that violet light PDT was able to bleach melanin in melanotic tumors and therefore increase their sensitivity to red light PDT. The hypericin photocytoxicity toward pigmented UCT MEL-1 melanoma cells was found to be lower than that in unpigmented A375 melanoma, due to the protective properties of melanin on ROS (8). Recently, Mroz et al (9) reported the effectiveness of Photofrin and bacteriochlorin 3 in relation to the melanin content of B16 melanoma cells. The effectiveness of Photofrin to induce cell death was completely abolished in B16F10 cells with a high pigment content.

Many pathways have been discovered whereby mammalian cells execute cell death in response to PDT with different photosensitizers (10). The porphyrin family molecules such as 5-ALA are localized in mitochondria. After PDT treatment they induce ROS generation as described in numerous studies $(6,11)$ and mainly produce a mitochondrial apoptosis with caspase activation. The moderate pan-caspase activity in pigmented B16F10 cells treated with $10 \mathrm{mM}$ 5-ALA was compatible with mitochondrial apoptosis with caspase activation such as described by Buytaert et al (5). Radzi et al (12) in the same-pigmented cells irradiated in the presence of indocyanine green observed a caspase-independent apoptosis. This is similar to the type of death that we observed in amelanotic variant B16G4F cells by 5-ALA.

Western blot analysis of p53 and PUMA (one of its transcriptional targets) confirmed that in the presence of melanin in the B16F10 melanoma cells a caspase-dependent apoptosis was recruited. Data support the contribution of a p53-regulated response to PDT in cells expressing wild-type p53 as described (13). In the amelanotic variant B16G4F, no p53 and PUMA signals were detected, suggesting that the mitochondrial pathway was not activated. Prasad et al (14) showed that in 7 patients with desmoplastic melanoma ( $0.1 \%$ of cutaneous melanoma) characterized by the presence of amelanotic invasive spindle cells, 6 patients had an aberrant or null p53 expression. The absence of p53 and PUMA expression with caspase-independent signaling was supported by 
results indicating a role of a p53-independent mechanism in response to PDT in p53-deficient cells (13). Both apoptosis and autophagy can occur after PDT (15), and recent studies described a non-apoptotic cell death associated with the induction of autophagy $(2,3,15,16)$.

In the B16F10 melanoma cell line our results showed that the absence of expression of an autophagic marker (beclin-1, LC3-I and LC3-II, cathepsin B) was in line with a mitochondrial caspase-dependent apoptosis. This mitochondrial death has been described in several melanoma cell lines treated with PDT and molecules of 5-ALA $(6,17)$. On the other hand, in the amelanotic variant B16G4F cells, PDT treatment with 5-ALA led to the activiation of autophagy as described using the same photosensitizer in non-pigmented cells such as skin keratinocytes (18), PC12 and CL1-0 cells (3). The recycling process can lead to cell death when cells attempt to recycle damaged constituents beyond their capacity for recovery or in apoptosis-incompetent cells $(15,19)$.

In summary, we demonstrated that 5-ALA and PDT with red illumination induced a differential susceptibility in melanoma cells based on the presence of melanin. In addition, mitochondrial apoptotic signaling was activated in the pigmented B16F10 cells, whereas an autophagic process leading to caspase-independent cell death occurred in amelanotic B16G4F cells. Further studies may confirm the attribution of PDT sensitivity of B16G4F cells to the lack of melanin and/ or a p53 gene mutation.

\section{Acknowledgements}

We would like to thank Dr Cornelia Wilson-Whelan (University of Limoges) for the critical language editing of the manuscript, Tan Ouk and Anne-Sophie Rigaudeau for their technical advice.

\section{References}

1. Choudhary S, Nouri K and Elsaie ML: Photodynamic therapy in dermatology: a review. Lasers Med Sci 24: 971-980, 2009.

2. Davids LM and Kleemann B: Combating melanoma: the use of photodynamic therapy as a novel, adjuvant therapeutic tool. Cancer Treat Rev 37: 465-475, 2011.
3. Ji HT, Chien LT, Lin YH, et al: 5-ALA-mediated photodynamic therapy induces autophagic cell death via AMP-activated protein kinase. Mol Cancer 9: 91, 2010.

4. Zuliani T, Duval R, Jayat C, et al: Sensitive and reliable JC-1 and TOTO-3 double staining to assess mitochondrial transmembrane potential and plasma membrane integrity: interest for cell death investigations. Cytometry A 54: 100-108, 2003.

5. Buytaert E, Dewaele M and Agostinis P: Molecular effectors of multiple cell death pathways initiated by photodynamic therapy. Biochim Biophys Acta 1776: 86-107, 2007.

6. Krestyn E, Kolarova H, Bajgar R, et al: Photodynamic properties of ZnTPPS(4), ClAlPcS(2) and ALA in human melanoma G361 cells. Toxicol In Vitro 24: 286-291, 2010.

7. Ma LW, Nielsen KP, Iani V, et al: A new method for photodynamic therapy of melanotic melanoma - effects of depigmentation with violet light photodynamic therapy. J Environ Pathol Toxicol Oncol 26: 165-172, 2007.

8. Davids LM, Kleemann B, Kacerovská D, et al: Hypericin phototoxicity induces different modes of cell death in melanoma and human skin cells. J Photochem Photobiol B 91: 67-76, 2008.

9. Mroz P, Huang YY, Szokalska A, et al: Stable synthetic bacteriochlorins overcome the resistance of melanoma to photodynamic therapy. FASEB J 24: 3160-3170, 2010.

10. Robertson CA, Evans DH and Abrahamse H: Photodynamic therapy (PDT): a short review on cellular mechanisms and cancer research applications for PDT. J Photochem Photobiol B 96: 1-8, 2009.

11. Kästle M, Grimm S, Nagel R, et al: Combination of PDT and inhibitor treatment affects melanoma cells and spares keratinocytes. Free Radic Biol Med 50: 305-312, 2011.

12. Radzi R, Osaki T, Tsuka T, et al: Photodynamic hyperthermal therapy with indocyanine green (ICG) induces apoptosis and cell cycle arrest in B16F10 murine melanoma cells. J Vet Med Sci 74: 545-551, 2011.

13. Zawacka-Pankau J, Krachulec J, Grulkowski I, et al: The p53-mediated cytotoxicity of photodynamic therapy of cancer: recent advances. Toxicol Appl Pharmacol 232: 487-497, 2008.

14. Prasad ML, Patel SG and Busam KJ: Primary mucosal desmoplastic melanoma of the head and neck. Head Neck 26: 373-377, 2004.

15. Kessel D and Oleinick NL: Initiation of autophagy by photodynamic therapy. Methods Enzymol 431: 1-16, 2009.

16. François A, Marchal S, Guillemin F, et al: mTHPC-based photodynamic therapy induction of autophagy and apoptosis in cultured cells in relation to mitochondria and endoplasmic reticulum stress. Int J Oncol 39: 1537-1543, 2011.

17. Tsai T, Ji HT, Chiang PC, et al: ALA-PDT results in phenotypic changes and decreased cellular invasion in surviving cancer cells. Lasers Surg Med 41: 305-315, 2009.

18. Silva JN, Galmiche A, Tomé JP, et al: Chain-dependent photocytotoxicity of tricationic porphyrin conjugates and related mechanisms of cell death in proliferating human skin keratinocytes. Biochem Pharmacol 80: 1373-1385, 2010.

19. Reiners JJ, Agostinis P, Berg K, et al: Assessing autophagy in the context of photodynamic therapy. Autophagy 6: 7-18, 2010. 